\title{
THREE-MANIFOLDS AND KÄHLER GROUPS
}

\author{
D. KOTSCHICK
}

\begin{abstract}
We give a simple proof of a result originally due to Dimca and Suciu [6]: a group that is both Kähler and the fundamental group of a closed three-manifold is finite. We also prove that a group that is both the fundamental group of a closed three-manifold and of a non-Kähler compact complex surface is $\mathbb{Z}$ or $\mathbb{Z} \oplus \mathbb{Z}_{2}$.
\end{abstract}

\section{INTRODUCTION}

In the late 1980s the study of Kähler groups, that is, fundamental groups of closed Kähler manifolds, took off in spectacular fashion. While restrictions on such groups were previously known because of Hodge theory and because of rational homotopy theory, several deep new results were proved around 1988. I will only recall two of them here. These and many other results on Kähler groups are discussed in detail in [2].

Firstly, generalising partial results of Johnson and Rees [11], Gromov proved:

Theorem 1 (Gromov [7]). A Kähler group does not split as a nontrivial free product.

Secondly, building on work of Siu, Sampson and others, Carlson and Toledo proved:

Theorem 2 (Carlson-Toledo [5]). No fundamental group of a closed real hyperbolic n-manifold with $n \geq 3$ is a Kähler group.

When these results were proved, several people, including Donaldson and Goldman, noticed the contrast between Kähler groups on the one hand and three-manifold groups on the other: the latter are closed under free products, and, according to Thurston, most three-manifolds with freely indecomposable fundamental group are hyperbolic. Moreover, a case by case check of the Thurston geometries as explained in [23] shows the following: closed three-manifolds carrying one of the geometries $S^{2} \times \mathbb{R}, \mathbb{H}^{2} \times \mathbb{R}, \mathbb{R}^{3}$ or Sol ${ }^{3}$ have virtually odd first Betti number, and so their fundamental groups cannot be Kähler. Moreover, closed three-manifolds carrying one of the geometries $\mathrm{Nil}^{3}$ or $\mathrm{SL}_{2}(\mathbb{R})$ have virtually positive first Betti numbers with trivial cup product from $H^{1}$ to $H^{2}$. Their fundamental groups cannot be Kähler by the Hard Lefschetz Theorem. Now, the only Thurston geometry that has not been excluded is $S^{3}$, where every fundamental group is finite. Since all finite groups are Kähler, it was natural to expect that the intersection of three-manifold groups with the Kähler groups should consist exactly of the finite groups appearing as fundamental groups of three-manifolds with geometry $S^{3}$. The obstacle to turning this expectation into a theorem, indeed a corollary of the above Theorems 1 and 2, came from three-manifolds with a non-trivial JSJ decomposition along incompressible tori. While one could imagine that those manifolds containing

Date: February 10, 2011; (c) D. Kotschick 2010.

2000 Mathematics Subject Classification. primary 32Q15, 57M05; secondary 14F35, 32J15, 57M50. 
at least some hyperbolic piece might yield to a generalisation of the harmonic map techniques of Carlson and Toledo [5] 1 , the case of graph manifolds seemed intractable.

Twenty years ago one thought about such questions modulo Thurston's geometrisation conjecture. Since this has now been proved by Perelman [21, 22, 13, 19], an unconditional result can finally be obtained. Indeed, Dimca and Suciu recently proved:

Theorem 3 (Dimca-Suciu [6]). Assume that a group $\Gamma$ is the fundamental group both of a closed Kähler manifold and of a closed three-manifold. Then $\Gamma$ is finite, and, therefore, a finite subgroup of $O(4)$.

Once one proves $\Gamma$ to be finite, it follows from Perelman's work [21, 22, 13, 19] that $\Gamma$ is a finite subgroup of $O(4)$ acting freely on $S^{3}$. Note that by a classical construction due to Serre, every finite group is the fundamental group of a smooth complex projective variety, hence a closed Kähler manifold. By the Lefschetz hyperplane theorem one may assume this variety to be a surface.

To me, a surprising aspect of the proof given by Dimca and Suciu is that it does not follow the above outline at all, and makes little use of the Thurston approach to three-manifolds. In fact, their proof does not use Theorems 1 and 2 . Instead, they consider separately the cases of trivial and of nontrivial first Betti number. If the first Betti number of the fundamental group of a closed oriented three-manifold is positive, then they prove it is not Kähler using a lot of machinery of a very different sort: characteristic and resonance varieties, Catanese's approach to the SiuBeauville theorem, a commutative algebra result of Buchsbaum-Eisenbud, .... Then, for the case of zero first Betti number, Dimca and Suciu appeal to results of Reznikov and Fujiwara pertaining to Kazhdan's property $T$. It is only at this point that their proof depends on geometrisation via Fujiwara's arguments.

The present paper arose from my attempt to understand the argument of Dimca and Suciu [6]. From their treatment of the positive Betti number case I extracted the following strategy for obtaining a contradiction: If $\Gamma$ has positive first Betti number and is both the fundamental group of a closed oriented three-manifold and of a closed Kähler manifold, then $H^{1}(\Gamma ; \mathbb{R})$ comes from a complex curve. Therefore all cup products of classes in $H^{1}(\Gamma ; \mathbb{R})$ also come from a curve, and this is incompatible with three-dimensional Poincaré duality.

One can actually implement this strategy in several different ways to prove Theorem 3 . Here I will give quite a different implementation from that in [6], leading to a quick proof of the following:

Theorem 4. If $\Gamma$ is a group with $b_{1}(\Gamma)>0$ whose real cohomology algebra $H^{*}(\Gamma ; \mathbb{R})$ satisfies 3-dimensional oriented Poincaré duality, then $\Gamma$ is not a Kähler group.

To put this into perspective, recall that many Kähler groups are Poincaré duality groups (of even dimension), cf. [11, 25, 14]. Also recall that, for every $k \geq 3$, Toledo [25] constructed examples of Kähler groups of cohomological dimension $2 k-1$. Moreover, his examples are duality (though not Poincaré duality) groups.

Of course, to exclude a group from being a Kähler group, it is enough that some finite index subgroup satisfy the assumptions of Theorem 4, Thus Theorem 4 immediately gives:

Corollary 5. Let $M$ be a closed aspherical three-manifold. If $M$ has a finite orientable covering that is not an $\mathbb{R}$-homology sphere, then $\pi_{1}(M)$ is not a Kähler group.

\footnotetext{
${ }^{1}$ A first step in this direction was soon taken by Hernández-Lamoneda, although his paper [10] was only published much later.
} 
Theorem 4 is more general than the Corollary because not every group whose real cohomology satisfies 3-dimensional Poincaré duality is the fundamental group of an aspherical three-manifold. This issue is related to the three-dimensional Borel conjecture; see Problem 3.77 on Kirby's problem list [12].

Corollary 5 proves most of Theorem 3 , since it handles not only manifolds with a nontrivial JSJ decomposition, but also gives a uniform treatment of geometric cases that no longer need to be checked case by case, so we obtain quite a simple proof of Theorem 3 for groups with virtually positive first Betti number. Using Perelman's geometrisation theorem, the case of first Betti number zero can actually be reduced to Theorem 2. In Section 2 below we first prove Theorem 4 , and then spell out the resulting straightforward proof of Theorem 3, avoiding the difficult arguments of Dimca-Suciu [6], and the appeals to the works of Reznikov and Fujiwara. Like the original proof of [6], the proof of Theorem 3 given here uses geometrisation only to handle the case of trivial (virtual) first Betti number.

Using the Kodaira classification of non-Kähler complex surfaces we shall also prove the following:

Theorem 6. Assume that a group $\Gamma$ is the fundamental group both of a closed complex surface $S$ and of a closed three-manifold. Then either $\Gamma$ is a finite subgroup of $O(4)$ and $S$ is a Kähler surface, or $\Gamma$ is $\mathbb{Z}$ or $\mathbb{Z} \oplus \mathbb{Z}_{2}$ and $S$ is a surface of class $V I I$.

This is interesting since in real dimension 6 every finitely presentable group is the fundamental group of a compact complex manifold, as proved by Taubes [24]. Thus, for fundamental group questions, complex surfaces are at the watershed between curves and the unrestricted case of complex three-folds, just like three-manifolds are at the watershed between real surfaces and the case of four-manifolds, where all finitely presentable groups appear.

\section{PROOFS}

Proof of Theorem 4. Suppose for a contradiction that $X$ is a closed Kähler manifold with fundamental group $\Gamma$, and let $\alpha_{X}: X \longrightarrow T^{b_{1}(\Gamma)}$ be its Albanese map. By the universal property of classifying maps, $\alpha_{X}$ factors up to homotopy into a composition

$$
X \stackrel{c_{X}}{\longrightarrow} B \Gamma \stackrel{a}{\longrightarrow} B \mathbb{Z}^{b_{1}(\Gamma)}=T^{b_{1}(\Gamma)},
$$

where $c_{X}$ is the classifying map of the universal covering of $X$. One concludes that $\alpha_{X}^{*}=c_{X}^{*} \circ a^{*}$ is trivial in real cohomology of degree $>3$ because $B \Gamma$ has no such cohomology, and so the image of $\alpha_{X}$ cannot have complex dimension 2 or more. Thus the image of $\alpha_{X}$ is a complex curve $C$.

It is well known, and easy to see, that a one-dimensional Albanese image must be smooth, and of course it has positive genus. Thus the Albanese map $\alpha_{X}$ factors as

$$
X \stackrel{c_{X}}{\longrightarrow} B \Gamma \stackrel{\hat{a}}{\longrightarrow} C \text {. }
$$

All the maps above induce isomorphisms in degree one cohomology. Moreover, $\alpha_{X}^{*}$ is nontrivial in degree 2 cohomology, and so the same is true for $\hat{a}^{*}$. However, there is no class in $H^{1}(\Gamma ; \mathbb{R})$ that has a nontrivial cup product with the image of $\hat{a}^{*}$ in $H^{2}(\Gamma ; \mathbb{R})$, since this cup product comes from $C$, which has real dimension $=2$. This contradicts the assumption that $\Gamma$ satisfies 3 -dimensional Poincaré duality.

Proof of Theorem 3. We need to show that an infinite three-manifold group $\Gamma$ cannot be Kähler. Since finite coverings of Kähler manifolds are Kähler, we only need to exclude some finite index 
subgroup of $\Gamma$, and so three-manifolds can be replaced by their finite coverings. In particular we may assume that all three-manifolds are orientable.

We may restrict our attention to three-manifolds that are prime in the sense of being indecomposable under connected sums, since a nontrivial free product is never a Kähler group by Theorem 1. Such a prime three-manifold is either $S^{1} \times S^{2}$, or is aspherical, cf. [18]. Since a Kähler group cannot be infinite cyclic, we are reduced to the consideration of aspherical three-manifolds, so that, for all 3-manifolds with positive (virtual) first Betti number, Theorem 3 follows from Corollary 5 , which in turn follows from Theorem 4 proved above.

To complete the proof of Theorem 3 it remains to deal with groups with vanishing first Betti number. Thus consider a closed oriented aspherical three-manifold $M$ with infinite fundamental group $\Gamma$ having $b_{1}(\Gamma)=0$. If $M$ contains an incompressible torus, then by a result of Luecke [17], see also [15], $M$ has a finite covering with positive first Betti number, so that Corollary 5 applied to this covering shows that $\Gamma$ is not Kähler. Thus we are left with the case of an aspherical $M$ that contains no incompressible torus. Such manifolds are hyperbolic by the work of Perelman [21, 22, 13], and fundamental groups of hyperbolic three-manifolds are never Kähler by Theorem 2 .

Proof of Theorem 6. Suppose that $\Gamma$ is the fundamental group of both a compact complex surface $S$ and a closed three-manifold $M$. As before we may assume $M$ to be orientable.

If $S$ is Kähler, then $\Gamma$ is finite by Theorem 3 . Conversely, if $\Gamma$ is finite, then the first Betti number of $S$ vanishes, and so $S$ is Kählerian, cf. [4].

If $S$ is not Kählerian, then its first Betti number is odd, see again [4]. We now use the EnriquesKodaira classification to conclude that either $S$ is properly elliptic with $b_{1}(S) \geq 3$, or $S$ is of class $V I I$ with $b_{1}(S)=1$, cf. [3, 20]. In the first case $\Gamma$ is freely indecomposable and is a Poincare duality group of dimension 4 by results of Kodaira described in [2, Section 3 of Ch. 1]. In the second case, it is known only that $\pi_{1}(S)$ cannot split into $\Gamma_{1} \star \Gamma_{2}$ with both $\Gamma_{i}$ containing proper subgroups of finite index; see [2, Thm. 1.35]. However, since three-manifold groups are residually finite [9] 2 , this is enough to conclude that in our case, where $\pi_{1}(S)=\Gamma=\pi_{1}(M), \Gamma$ is indeed freely indecomposable.

Thus we may assume that $M$ is prime. If it is aspherical, then $\Gamma$ is a three-dimensional Poincaré duality group. This means that $\Gamma$ is not the fundamental group of a properly elliptic surface with $b_{1}(S) \geq 3$ since those groups are four-dimensional Poincare duality groups. If $\Gamma$ is the fundamental group of a class $V I I$ surface, then we have $b_{1}(\Gamma)=1$, and, by Poincaré duality on $M, b_{2}(\Gamma)=1$. Under the classifying map of the universal covering of $S, H^{2}(\Gamma ; \mathbb{R})$ injects into $H^{2}(S ; \mathbb{R})$, where it becomes an isotropic subspace for the cup product for dimension reasons. (Its cup square comes from the three-dimensional $M$.) Thus the intersection form of $S$ would have to be indefinite, which contradicts the known fact that the intersection forms of class VII surfaces are negative definite; see [2, Lemma 1.45].

Thus we are left to consider the case of an $M$ that is prime but not aspherical. This means that $M$ is $S^{1} \times S^{2}$ if it is orientable; cf. [18]. However, for a nonorientable $M$ we could also have the nontrivial $S^{2}$-bundle over $S^{1}$, also with fundamental group $\mathbb{Z}$, and $S^{1} \times \mathbb{R} P^{2}$, with fundamental group $\mathbb{Z} \oplus \mathbb{Z}_{2}$; cf. [23]. Both $\mathbb{Z}$ and $\mathbb{Z} \oplus \mathbb{Z}_{2}$ occur as fundamental groups of Hopf surfaces. Conversely, every surface with one of these fundamental groups is of class VII; cf. [3, 20]. This completes the proof of Theorem 6 .

\footnotetext{
${ }^{2}$ The reference [9] treats only manifolds satisfying Thurston's geometrisation conjecture. By Perelman's work [21, 22, 13] this is not a restriction.
} 


\section{DISCUSSION}

3.1. Avoiding the use of Theorem 1, In the proof of Theorem 3 in Section 2, I found it most straightforward to reduce to the consideration of prime three-manifolds by using Gromov's result on free products, stated as Theorem 1 in the introduction. However, one can completely bypass the use of Theorem 1, as we now explain.

Lemma 7. Assume that $\Gamma_{1}$ and $\Gamma_{2}$ each have a non-trivial finite quotient $f_{i}: \Gamma_{i} \longrightarrow Q_{i}$. Then their free product $\Gamma_{1} \star \Gamma_{2}$ has a finite index subgroup with odd first Betti number.

Proof. Consider the induced homomorphism $f: \Gamma_{1} \star \Gamma_{2} \longrightarrow Q_{1} \times Q_{2}$. By the Kurosh subgroup theorem, its kernel is of the form $F_{k} \star \Gamma$, where $F_{k}$ is a free group of rank $k=\left(\left|Q_{1}\right|-1\right)\left(\left|Q_{2}\right|-1\right)$, and $\Gamma$ is a free product of copies of the kernels of the $f_{i}$. For a finite quotient $g: F_{k} \longrightarrow Q$ of order $d$ we consider the kernel $\Delta$ of $\bar{g}: F_{k} \star \Gamma \longrightarrow Q$, where $\bar{g}$ restricts to $F_{k}$ as $g$ and is trivial on $\Gamma$. Then $\Delta$ is isomorphic to $F_{l} \star \Gamma \star \ldots \star \Gamma$ with $d$ copies of $\Gamma$ appearing, and $l=1+d(k-1)$. Thus $\Delta \subset \Gamma_{1} \star \Gamma_{2}$ is a finite index subgroup with

$$
b_{1}(\Delta)=l+d \cdot b_{1}(\Gamma)=1+d \cdot\left(k-1+b_{1}(\Gamma)\right) .
$$

Choosing $d$ to be even, we have found the desired subgroup.

Since three-manifold groups are residually finite [9], we have the following:

Corollary 8. If $M$ is a non-prime three-manifold, then it has a finite covering with odd first Betti number.

At the expense of appealing to residual finiteness, we can use this Corollary in place of Theorem 1 to exclude non-prime manifolds from consideration in the proof of Theorem 3 , More generally, without restricting to three-manifold groups, Lemma 7 tells us that an arbitrary free product whose free factors admit finite quotients cannot be a Kähler group. This is exactly the special case of Theorem 1 originally proved by Johnson and Rees [11]. Indeed our proof of the Lemma is a simplification of the argument in [11].

3.2. The necessity to discuss $\mathbb{R}$-homology spheres. In the proof of Theorem 3 it was necessary to consider separately the case of groups with zero first Betti number. This step would be superfluous, if it were known that every closed three-manifold has a finite covering with positive first Betti number. If such a statement were available, then one would not need Theorem 2 for the proof of Theorem 3 given here.

Apparently the question of whether every closed three-manifold with infinite fundamental group has virtually positive first Betti number was raised long ago by Waldhausen, Thurston, and others; see Problems 3.2 and 3.50 in Kirby's problem list [12] and the references given there. Curiously, those references do not include [8, 17] and other papers quoted in [8], all of which contain a wealth of information about this problem. In any case, this problem seems to be still open.

3.3. The second Betti number of infinite Kähler groups. Carlson and Toledo have asked whether an infinite Kähler group has virtually positive second Betti number ${ }^{3}$. If this were known to be true, then, because of three-dimensional Poincaré duality, we would not have to consider $\mathbb{R}$-homology 3 -spheres in the proof of Theorem 3 . Moreover, we would not need to use geometrisation, and we would not need Theorem 2] either! We refer to the paper of Klingler [14] for a recent discussion of this question of Carlson and Toledo.

\footnotetext{
${ }^{3}$ The original reference for their question is Section 18.16 in [16], where only a more specific version is formulated.
} 
Unfortunately, a slight misstatement occurs in [2, Prop. 3.44 (i)], which implicitly asserts a positive answer to the question of Carlson and Toledo. The statement $b_{2}\left(\pi_{1}(X)\right) \geq 1$ there should be replaced by $b_{2}(X) \geq 1$ (which is trivial). The Proposition in question was proved by Amorós [1], whose paper does not contain the misstatement.

\section{REFERENCES}

1. J. Amorós, On the Malcev completion of Kähler groups, Comment. Math. Helv. 71 (1996), 192-212.

2. J. Amorós, M. Burger, K. Corlette, D. Kotschick and D. Toledo, Fundamental Groups of Compact Kähler Manifolds, Mathematical Surveys and Monographs, Vol. 44, Amer. Math. Soc., Providence, R.I. 1996.

3. W. Barth, C. Peters and A. Van de Ven, Compact Complex Surfaces, Springer-Verlag, Berlin 1984.

4. N. Buchdahl, On compact Kähler surfaces, Ann. Inst. Fourier 49 (1999), 287-302.

5. J. A. Carlson and D. Toledo, Harmonic mapping of Kähler manifolds to locally symmetric spaces, Publ. Math. I.H.E.S. 69 (1989), 173-201.

6. A. Dimca and A. I. Suciu, Which 3-manifold groups are Kähler groups?, J. Eur. Math. Soc. 11 (2009), $521-528$.

7. M. Gromov, Sur le groupe fondamental d'une variété kählérienne, C. R. Acad. Sci. Paris Sér. I Math. 308 (1989), 67-70.

8. J. Hempel, Virtually Haken manifolds, in Combinatorial methods in topology and algebraic geometry (Rochester, N.Y., 1982), 149-155, Contemp. Math., 44, Amer. Math. Soc., Providence, RI, 1985.

9. J. Hempel, Residual finiteness for 3-manifolds, in Combinatorial groups theory and topology, ed. S. M. Gersten and J. R. Stallings, Annals of Math. Studies vol. 111, Princeton Univ. Press 1987.

10. L. Hernández-Lamoneda, Non-positively curved 3-manifolds with non-Kähler $\pi_{1}$, C. R. Acad. Sci. Paris Sér. I 332 (2001), 249-252.

11. F. E. A. Johnson and E. G. Rees, On the fundamental group of a complex algebraic manifold, Bull. London Math. Soc. 19 (1987), 463-466.

12. R. Kirby, Problems in low-dimensional topology, in Geometric Topology, vol. 2, ed. W. H. Kazez, AMS/IP Studies in Advanced Mathematics Vol. 2 part 2, American Mathematical Society and International Press 1997.

13. B. Kleiner and J. Lott, Notes on Perelman's papers, Geom. Topol. 12 (2008), 2587-2855.

14. B. Klingler, Kähler groups and duality, Preprint arXiv:1005.2836v1 [math.GR] 17 May 2010.

15. S. Kojima, Finite covers of 3-manifolds containing essential surfaces of Euler characteristic $=0$, Proc. Amer. Math. Soc. 101 (1987), 743-747.

16. J. Kollár, Shafarevich maps and automorphic forms, Princeton Univ. Press, Princeton, NJ 1995.

17. L. Luecke, Finite covers of 3-manifolds containing essential tori, Trans. Amer. Math. Soc. 310 (1988), $381-391$.

18. J. W. Milnor, A unique decomposition theorem for 3-manifolds, Amer. J. Math. 84 (1962), 1-7.

19. J. W. Morgan and G. Tian, Ricci flow and the Poincaré conjecture, Amer. Math. Soc. and Clay Math. Institute 2007.

20. I. Nakamura, Towards classification of non-Kählerian complex surfaces, Sugaku Exp. 2 (1989), $209-229$.

21. G. Perelman, The entropy formula for the Ricci flow and its geometric applications, Preprint arXiv:math/ 0211159v1 [math.DG] 11 Nov 2002.

22. G. Perelman, Ricci flow with surgery on three-manifolds, Preprint arXiv:math/0303109v1 [math.DG] 10 Mar 2003.

23. P. Scott, The geometries of 3-manifolds, Bull. London Math. Soc. 15 (1983), 401-487.

24. C. H. Taubes, The existence of anti-self-dual conformal structures, J. Differential Geometry 36 (1992), $163-253$.

25. D. Toledo, Examples of fundamental groups of compact Kähler manifolds, Bull. London Math. Soc. 22 (1990), 339-343.

Mathematisches Institut, LMU MÜnChen, Theresienstr. 39, 80333 MünChen, GeRMAnY

E-mail address: dieter@member.ams . org 